\title{
Disfunção erétil associada à prática de atividade física e qualidade de vida de idosos
}

\section{Erectile dysfunction associated to physical activity and quality of life in older people}

Patricia Heck', Camila da Cruz Ramos de Araujo', Joris Pazin', Tayse Seemann', Paulo André Medeiros de Oliveira', Zenite Machado', Adriana Coutinho de Azevedo Guimarães²

\begin{abstract}
Resumo
Objetivou-se analisar a relação entre a atividade física e a qualidade de vida com a disfunção erétil de 121 idosos participantes de grupos de convivência cadastrados na prefeitura de

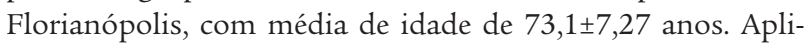
cou-se um questionário em forma de entrevista contendo: a) identificação pessoal; b) situação socioeconômica; c) Nível de atividade física (IPAQ- versão longa adaptada para idosos); d) Qualidade de vida: WHOQOL-bref; e) Índice Internacional de Função Erétil. Verificou-se prevalência elevada de idosos com disfunção erétil (63\%), sendo $18 \%$ suave, $8 \%$ moderada e $36 \%$ severa. Os idosos com e sem disfunção erétil foram suficientemente ativos (67\%). Em relação a qualidade de vida, aqueles idosos com disfunção erétil apresentaram escores inferiores nos domínios Psicológico e Social, quando comparados aos sem disfunção. Houve alta prevalência de disfunção erétil e essa variável parece diminuir a percepção de qualidade de vida nos domínios psicológico e social.
\end{abstract}

\section{Palavras-chave}

Disfunção sexual; Atividade motora; Idosos.

\begin{abstract}
This study aimed to analyze how physical activity and quality of life are related to erectile dysfunction in 121 older people, mean age $73.1 \pm 7.27$ years, from senior centers that are registered at the Florianópolis City Hall. Interviews were conducted using a questionnaire consisting of: a) Personal identification; b) Socioeconomic status; c) Physical activity level (IPAQ - long version adapted to older people); d) Quality of life: WHOQOL-bref; e) International index of erectile function (IIEF). It was found a high prevalence of older people who suffer from erectile dysfunction (63\%), of which $18 \%$ were classified as mild, $8 \%$ as moderate and $36 \%$ as severe. Older people with erectile dysfunction as well as the ones without it were classified as sufficiently active (67\%). As to quality of life, those older people who suffer from erectile dysfunction showed lower scores in the psychological and social domains when compared to the ones who don't suffer from the dysfunction. Most of the research sample presented severe and moderate erectile dysfunction. These variables seem to decrease the perception of quality of life in the psychological and social domains.
\end{abstract}

\section{Keywords}

Sexual Dysfunction; Motor Activity; Aged.

\section{Introdução}

Os homens vivem menos e com menor qualidade se comparados às mulheres, procuram menos os serviços de atenção primária à saúde e têm menor participação em atividades que influenciam comportamentos de saúde ${ }^{1,2}$ Assim, estão mais sujeitos ao desenvolvimento de inúmeras doenças, dentre elas as doenças sexuais, como a disfunção erétil (DE). Homens com histórico de câncer de próstata, hipertensão arterial, tabagismo, obesidade, diabetes e depressão, também têm risco aumentado para $\mathrm{DE}^{3,4}$. Ainda,

1 Centro de Ciências da Saúde e do Esporte (CEFID), Universidade do Estado de Santa Catarina, (UDESC), Florianópolis, SC, Brasil.

2 Departamento de Educação Física e do Programa de Pós-Graduação em Ciências do Movimento Humano do Centro de Ciências da Saúde e do Esporte (CEFID), Universidade do Estado de Santa Catarina (UDESC), Florianópolis, SC, Brasil. homens com DE grave apresentam maior risco de ter síndrome metabólica ${ }^{5}$.

No Brasil, um estudo realizado em homens com média de idade de 37,6 anos, constatou que 45,1\% apresentaram algum nível de DE, sendo $31,2 \%$ mínima, $12,2 \%$ moderada e $1,7 \%$ completa $^{3}$. Apesar de não acarretar risco de vida, a DE pode afetar o relacionamento interpessoal, comprometer o bem-estar e a qualidade de vida $(\mathrm{QV})$ masculina ${ }^{3}$.

Atualmente é possível observar avanços farmacológicos e cirúrgicos no tratamento da DE, o que desperta crescente interesse na população em geral e profissionais da saúde, gerando maior demanda de serviços clínicos especializados ${ }^{6}$. Entretanto, a literatura demonstra que além de tais métodos interventivos, a prática de 
atividade física (AF) tem sido apontada como tratamento importante para esta disfunção $\mathrm{o}^{7-8}$. Estudos apontam ainda, que a falta ou a prática limitada de $\mathrm{AF}$ podem ser preditores da $\mathrm{DE}^{7}$. A relação entre $\mathrm{AF}$ e $\mathrm{DE}$ poderia ser justificada pelos benefícios que a $\mathrm{AF}$ proporciona ao sistema cardiovascular, na redução de doenças vasculares, melhoria das funções endoteliais e aumento do fluxo sanguíneo em determinados leitos vasculares ${ }^{9}$, sendo que tais efeitos podem auxiliar na vascularização peniana ${ }^{10}$.

A melhora dos fatores da DE pode ser obtida por meio de alterações no estilo de vida, adoção de hábitos nutricionais saudáveis e incentivo a prática de $\mathrm{AF}^{10,11}$. Nesse sentido a AF e consequentemente uma boa condição física, podem ser caracterizadas como medidas preventivas e terapêuticas para o controle da DE e doenças associadas ${ }^{12}$. Em virtude da escassez de estudos que relacionam a pratica de $\mathrm{AF}, \mathrm{QV}$ e $\mathrm{DE}$, sua possível prevenção e tratamento das doenças relacionadas à $\mathrm{DE}$, objetivou-se analisar a relação entre a $\mathrm{DE}$ e a $\mathrm{AF}$ com $\mathrm{QV}$ em homens idosos de Florianópolis - Santa Catarina.

\section{Métodos}

O estudo descritivo exploratório com delineamento transversal foi composto por amostra de conveniência com 121 homens idosos, com média de idade de $71,3 \pm 7,27$ anos, participantes de Grupos de Convivência cadastrados na prefeitura municipal de Florianópolis/ SC. Os programas de atendimento ao idoso são grupos de convivência cadastrados e vinculados à prefeitura de Florianópolis. As atividades dos grupos são planejadas e executadas pelos coordenadores de cada grupo, e normalmente oferecem alongamentos, dança, ginástica, bingo e excursão.

Para participar do estudo os idosos deveriam pertencer ao sexo masculino, ter 60 anos ou mais de idade e participar ativamente dos Grupos de Convivência. Excluíram-se da pesquisa idosos com algum tipo de deficiência física e mental, nomeadamente: limitação na coordenação motora, surdez, dificuldade visual ou qualquer tipo de demência já diagnosticada, além de idosos com diagnóstico de câncer de próstata. Foram excluídos 10 idosos: sete autorrelaram câncer de próstata e três, segundo as esposas, não tinham condições mentais para responder ao questionário.

A coleta dos dados foi realizada em formato de entrevista individual, por meio de um questionário dividido em cinco partes:

Informações Gerais: caracterizou os participantes quanto à idade, estado conjugal, escolaridade, índice de massa corporal, nível econômico, presença de doenças clinicamente diagnosticadas, uso de medicamentos para o desempenho sexual, frequência de uso de medicamentos para desempenho sexual e consumo de tabaco e álcool.

O consumo de álcool foi verificado por meio do questionário AUDIT ${ }^{13}$, que consiste em um questionário composto de 10 questões sobre o uso recente, dependência e sintomas e problemas relacionados ao uso do álcool. Esse protocolo classifica o indivíduo como em baixo risco (0-7 pontos), uso de risco (8-15 pontos), uso nocivo (16-19 pontos) e provável dependência (20-40 pontos) ${ }^{14}$. Para melhor análise de dados, as categorias: uso de risco, uso nocivo e provável dependência, foram agrupadas na categoria risco.

Medidas Antropométricas: massa corporal e estatura (autorreferidos) para o cálculo do Índice de Massa Corporal - IMC (peso/estaturaª ${ }^{2}$. Para classificação do IMC foi utilizado o protocolo da World Health Organization ${ }^{15}$, ou seja, abaixo do peso (IMC<18,49); eutrofia (IMC 18,50 a 24,99); sobrepeso (IMC 25,0 a 29,99); e obesidade (IMC>30,0), sendo essa dividida em grau 1(IMC 30,0 a 34,99), grau 2 (IMC 35,0 a 39,99) e grau 3 (IMC > 40,0). Para efeitos estatísticos, as categorias abaixo do peso e eutrofia foram agrupadas em peso normal; e as categorias sobre- 
peso e obesidade, independente do grau, agrupadas em acima do peso.

Nível Econômico: segundo critério do $\mathrm{IBGE}^{16}$, que classifica a população em níveis econômicos A, B, C, D e E. A categorização é feita por meio da renda familiar média mensal, resultando em: classe A (acima de 20 Salários Mínimos); classe B (10 a 20 SM); classe C (4 a 10 SM); classe D (2 a 4 SM) e classe E (até 2 SM). Para análise dos dados, os estratos classe $\mathrm{A}$ e $\mathrm{B}$ foram agrupados em classe alta; o estrato classe $\mathrm{C}$ em classe média; e os estratos classe $\mathrm{D}$ e $\mathrm{E}$ em classe baixa.

Nível de Atividade Física: Questionário Internacional de Atividade Física (IPAQ), versão longa, validado por Benedetti e colaboradores ${ }^{17}$ e adaptado por Mazo e Bene$\operatorname{detti}^{18}$, que tem como objetivo mensurar as AF realizadas nos domínios do trabalho, transporte, atividades domésticas e lazer. No IPAQ adaptado foram agrupadas, na mesma questão, as perguntas referentes "aos dias" e "ao tempo" dos diferentes domínios da $\mathrm{AF}$ (quais os dias e qual o tempo numa semana típica e por 10 minutos contínuos). Para resposta, foi construído um quadro com os dias da semana e turnos. Neste quadro foi anotado o tempo (minutos e horas) gasto em AF moderada e/ ou vigorosa em cada dia da semana e turnos (manha, tarde e noite). Posteriormente os tempos foram somados para obter o gasto total semanal. E assim, foram classificados em insuficientemente ativos abaixo de 150 minutos semanais e suficientemente ativos (SA) igual ou acima de 150 minutos semanais de atividade física.

Qualidade de vida relacionada à saúde: A QV foi avaliada por meio do questionário abreviado "World Health Organization Quality of Life" (WHOQOL - bref), adaptado para Português ${ }^{19}$. Questionário é composto por 26 questões, das quais 24 abrangem o domínio físico (dor física, energia, locomoção, atividades da vida diária, tratamento médico, trabalho), psicológico (sentimentos positivos, concentração, autoestima, autoimagem, sentimentos negativos, espiritualidade), social (relações pessoais, suporte social, atividade sexual), e meio ambiente (segurança física, habitação, recursos financeiros, serviço de saúde, informações, lazer, ambiente físico e transporte,) e mais duas questões gerais sobre QV. As questões são respondidas por meio de uma escala tipo Likert, expressa por uma escala de intensidade (nada - extremamente), ou de capacidade (nada - completamente), ou de frequência (nunca - sempre), ou de avaliação (muito insatisfeito - muito satisfeito; muito ruim - muito bom). Os valores são computados em escores que variam de 0 a 100, sendo que quanto mais próximo de 100 melhor a QV.

Disfunção Erétil: foi avaliada por meio do Índice Internacional de Função Erétil (IIFE) ${ }^{20}$. É um questionário breve, fidedigno e amplamente utilizado ${ }^{21,22}$. O IIFE apresenta tradução e adaptação para a língua portuguesa por Ferraz e Ciconelli²3. O questionário é composto de 15 questões que avaliam cinco parâmetros da função sexual masculina: função erétil (6 questões), função orgásmica (duas questões), desejo sexual (duas questões), satisfação na relação sexual (três questões) e satisfação sexual global (duas questões). Para a determinação do grau da DE foi utilizada a soma das questões 1, 2, 3, 4, 5 e 15. Classificadas em cinco categorias: ausência (26-30), suave (22-25), suave para moderada (17-21), moderada (11-16) e severa (6-10). Sendo que indivíduos que apresentaram pontuação menor ou igual a 25 , foram considerados com $\mathrm{DE}^{24}$. Para fins estatísticos, a classificação suave para moderada e moderada, foram agrupadas em moderada, ou seja, na seguinte sequencia: suave, moderada e severa.

O projeto foi aprovado pelo Comitê de Ética em Pesquisa em Seres Humanos (CEPSH) da UDESC, protocolo no 723.796 em 21 de julho de 2014. Os idosos foram convidados a participar do estudo voluntariamente e assinaram o termo de consentimento livre e esclarecido, garantindo dessa forma os direitos dos pesquisados, conforme prescrito na Resolução 466/2012 do Conselho Nacional de 
Saúde. A partir deste deu-se início a coleta de dados, que durou 8 meses, e foi feita por 3 pesquisadores em dia e horário previamente agendado.

A análise estatística foi realizada por meio do pacote estatístico The Statistical Package for the Social Sciences (SPSS) - versão 20.0. Fez-se uso da estatística descritiva, mediante o cálculo de média, desvio padrão e distribuição de frequências. A normalidade dos dados foi verificada pelo teste de Komogorov Smirnov. Para a comparação das características sócio demográficas com a DE utilizou-se os testes Qui quadrado e Exato de Fischer. Empregou-se os testes U Mann-Whitney, Kruskall-Wallis e Post Hoc Dunn para comparar a QV com a DE. A relação entre a DE e o nível de AF foi mensurada por meio do Teste do Qui-quadrado e Exato de Fischer. O nível de significância estatística adotado foi de $95 \%$.

\section{Resultados}

Conforme os dados apresentados na tabela 1, os maiores percentuais em cada variável indicam que os idosos apresentaram sobrepeso (46,2\%), estudaram até o ensino fundamental (48,3\%), são casados (87,5\%), apresentam doenças $(61,2 \%)$, não utilizam medicamento para desempenho sexual (91,7\%), pertencem à classe econômica baixa (59,3\%), praticam $\mathrm{AF}(67,8)$, apresentam baixo risco de dependência alcoólica (97,5\%) e não fumam (93,4\%). Apesar de não haver associação significativa entre as variáveis, todos os percentuais foram mais elevados para os idosos com DE.

TABELA 1 - Características dos homens participantes do estudo. Florianópolis. 2015.

\begin{tabular}{|c|c|c|c|c|c|}
\hline & $n$ & IC95\% & $\begin{array}{c}\text { Apresenta DE } \\
\mathrm{n}(\%)\end{array}$ & $\begin{array}{c}\text { Não apresenta } \\
n(\%)\end{array}$ & Valor de $p$ \\
\hline IMC & 119 & & & & 0,238 \\
\hline Normal & & $0,27-0,45$ & $22(29,7)$ & $21(46,6)$ & \\
\hline Sobrepeso & & $0,37-0,55$ & $36(48,6)$ & $19(42,2)$ & \\
\hline Obesidade & & $0,11-0,25$ & $16(21,5)$ & $5(11,2)$ & \\
\hline Escolaridade & 120 & & & & 0,499 \\
\hline Ensino fundamental & & $0,39-0,58$ & $36(47,3)$ & $22(50,0)$ & \\
\hline Ensino médio & & $0,29-0,47$ & $31(40,7)$ & $16(36,4)$ & \\
\hline Superior & & $0,07-0,20$ & $9(12,0)$ & $6(13,6)$ & \\
\hline Estado conjugal & 120 & & & & 0,390 \\
\hline Casado & & $0,81-0,94$ & $68(89,5)$ & $37(84,1)$ & \\
\hline Solteiros/ viúvos/ separados & & $0,06-0,19$ & $8(10,5)$ & $7(15,9)$ & \\
\hline Doenças & 121 & & & & 0,475 \\
\hline Apresenta & & $0,52-0,70$ & $46(60,5)$ & $27(60)$ & \\
\hline Não apresenta & & $0,30-0,48$ & $30(39,5)$ & $18(40,0)$ & \\
\hline Medicamento para desempenho sexual & 121 & & & & $0,497^{*}$ \\
\hline Utiliza & & $0,03-0,13$ & $5(6,6)$ & $5(11,1)$ & \\
\hline Não utiliza & & $0,87-0,97$ & $71(93,4)$ & $40(88,9)$ & \\
\hline Alcool & 121 & & & & $0,689^{*}$ \\
\hline Baixo risco & & $0,94-1,00$ & $74(97,4)$ & $44(97,8)$ & \\
\hline Risco & & $0-0,06$ & $2(2,6)$ & $1(2,2)$ & \\
\hline Tabaco & 121 & & & & $0,125^{*}$ \\
\hline Fuma & & $0,02-0,12$ & $3(3,9)$ & $5(11,1)$ & \\
\hline Não fuma & & $0,88-0,98$ & $73(96,1)$ & $40(88,9)$ & \\
\hline Nível econômico & 118 & & & & $0,927^{*}$ \\
\hline Classe alta & & $0,05-0,16$ & $8(10,8)$ & $4(9,1)$ & \\
\hline Classe média & & $0,22-0,39$ & $21(28,4)$ & $15(34,1)$ & \\
\hline Classe baixa & & $0,50-0,68$ & $45(60,8)$ & $25(56,8)$ & \\
\hline
\end{tabular}

Teste do Qui-quadrado; Exato de Fischer* DE: disfunção erétil; IMC: Índice de Massa Corporal; AF: Atividade Física. 
Na tabela 2 observa-se que a maioria $(62,8 \%)$ dos homens apresenta DE e que desses, um número elevado apresenta a DE na sua forma mais severa $(38,8 \%)$.

TABELA 2 - Prevalência da DE em idosos. Florianópolis. 2015. (n=121)

\begin{tabular}{lcc}
\hline & $n$ & $(\%)$ \\
\hline DE & & \\
\hline Apresenta DE & 76 & 62,8 \\
\hline Não apresenta DE & 45 & 37,2 \\
\hline
\end{tabular}

DE: disfunção erétil.

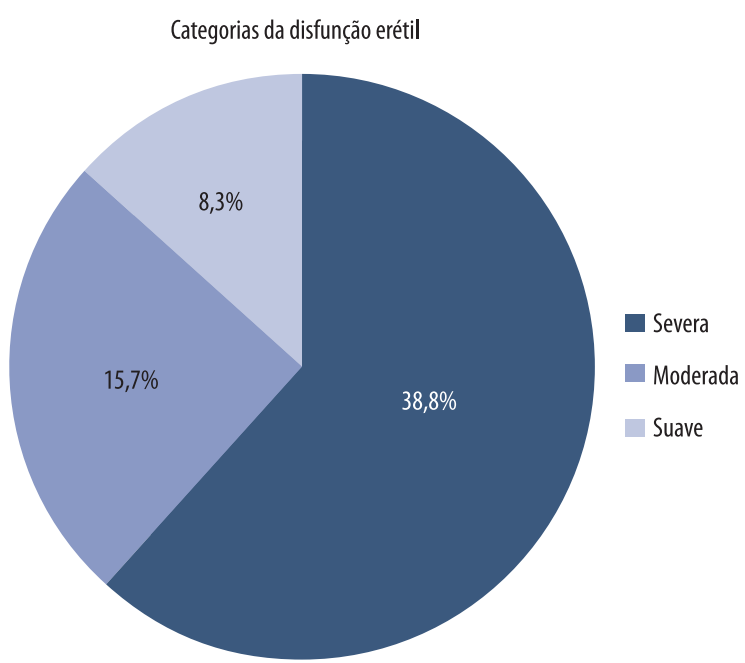

FIGURA 1 - Caracterização da disfunção em idosos.

O nível de AF dos participantes do estudo é apresentado na tabela 3, onde é possível observar que a maioria dos idosos são classificados como SA ( $\mathrm{n}=81)$, com destaque para aqueles com DE $(n=46)$. Dentre os idosos que possuem DE, quando categorizados de acordo com a severidade da doença, a maioria é classificada como SA, sendo $n=28$ da categoria severa, $n=13$ da moderada e $n=5$ da suave. Os resultados não apresentaram significância estatística.

TABELA 3 - Comparação do nível de atividade física com as categorias da DE. Florianópolis. 2015 $(n=121)$.

\begin{tabular}{|c|c|c|c|c|c|c|}
\hline & $\begin{array}{l}\text { Total } \\
\text { n (\%) }\end{array}$ & \multicolumn{2}{|c|}{$\begin{array}{c}\text { Apresenta DE } \\
\mathrm{n}(\%)\end{array}$} & \multicolumn{2}{|c|}{$\begin{array}{c}\text { Não apresenta DE } \\
n(\%)\end{array}$} & Valor de $p$ \\
\hline Nível de Atividade Física & & & & & & 0,051 \\
\hline Insuf. Ativo & $40(33,1)$ & \multicolumn{2}{|c|}{$30(39,5)$} & \multicolumn{2}{|c|}{$10(22,2)$} & \\
\hline \multirow[t]{2}{*}{ Suf. Ativo } & $81(66,9)$ & \multicolumn{2}{|c|}{$46(60,5)$} & \multicolumn{2}{|c|}{$35(77,8)$} & \\
\hline & $\begin{array}{l}\text { Total } \\
\text { n (\%) }\end{array}$ & $\begin{array}{l}\text { Severa } \\
\mathrm{n}(\%)\end{array}$ & $\begin{array}{c}\text { Moderada } \\
\text { n (\%) }\end{array}$ & $\begin{array}{l}\text { Suave } \\
\mathrm{n}(\%)\end{array}$ & $\begin{array}{c}\text { Sem DE } \\
n(\%)\end{array}$ & Valor de $\mathrm{p}$ \\
\hline Nível de Atividade Física & & & & & & $0,166^{*}$ \\
\hline Insuf. Ativo & $40(33,1)$ & $19(40,4)$ & $6(31,6)$ & $5(50,0)$ & $10(22,2)$ & \\
\hline Suf. Ativo & $81(66,9)$ & $28(59,6)$ & $13(68,4)$ & $5(50,0)$ & $35(77,8)$ & \\
\hline
\end{tabular}

Teste do Qui-quadrado; Exato de Fischer*. DE: disfunção erétil; AF: atividade física; Insuf. Ativo: insuficientemente ativo; Suf. Ativo: suficientemente ativo.

A QV dos idosos de acordo com a presença ou não de DE é apresentada na tabela 4, sendo que a maioria apresentou bons escores de QV, principalmente aqueles sem DE. Os homens com DE Severa e Moderada apresentaram pior qualidade de vida quando comparados aos sem DE nos domínios Psicológico $(\mathrm{p}=0,002)$ e Social $(\mathrm{p}<0,001)$. 
TABELA 4 - Comparação dos domínios da Qualidade de Vida com a DE. Florianópolis. 2015 ( $n=121$ ).

\begin{tabular}{|c|c|c|c|c|c|c|c|}
\hline Domínios da Qualidade de Vida & Total & \multicolumn{2}{|c|}{ Apresenta DE } & \multicolumn{2}{|c|}{ Não apresenta DE } & \multicolumn{2}{|l|}{ Valor de $p$} \\
\hline Físico & $67 \pm 11$ & \multicolumn{2}{|c|}{$66 \pm 12$} & \multicolumn{2}{|c|}{$68 \pm 9$} & \multicolumn{2}{|l|}{0,188} \\
\hline Psicológico & $70 \pm 9$ & \multicolumn{2}{|c|}{$68 \pm 9$} & \multicolumn{2}{|c|}{$73 \pm 7$} & \multicolumn{2}{|l|}{0,001} \\
\hline Social & $80 \pm 14$ & \multicolumn{2}{|c|}{$75 \pm 13$} & \multicolumn{2}{|c|}{$87 \pm 11$} & \multicolumn{2}{|l|}{0,000} \\
\hline Ambiental & $79 \pm 10$ & \multicolumn{2}{|c|}{$79 \pm 10$} & \multicolumn{2}{|c|}{$80 \pm 11$} & \multicolumn{2}{|l|}{0,586} \\
\hline Domínios da Qualidade de Vida & Total & Severa & Moderada & Suave & Sem DE & Valor de $p$ & Post hoc \\
\hline Físico & $67 \pm 11$ & $68 \pm 13$ & $62 \pm 11$ & $68 \pm 12$ & $68 \pm 9$ & 0,327 & - \\
\hline Psicológico & $70 \pm 9$ & $67 \pm 8$ & $66 \pm 10$ & $73 \pm 11$ & $73 \pm 7$ & 0,002 & $D>B, A$ \\
\hline Social & $80 \pm 14$ & $75 \pm 13$ & $76 \pm 12$ & $77 \pm 17$ & $87 \pm 11$ & $<0,001$ & $D>B, A$ \\
\hline Ambiental & $79 \pm 10$ & $78 \pm 10$ & $79 \pm 12$ & $81 \pm 8$ & $80 \pm 11$ & 0,902 & - \\
\hline
\end{tabular}

Nota: Para a DE dicotômica foi utilizado o Teste U Many Whitney, e para a Severidade da DE foi utilizado o Teste Kruskall-Wallis e Post Hoc Dunn. DE: disfunção erétil. Grupo A = Severa; Grupo B = Moderada; Grupo C = Suave; Grupo D = Sem DE.

\section{Discussão}

Ao analisar a relação entre a $\mathrm{DE}$ com a $\mathrm{AF}$ e $\mathrm{QV}$ em homens idosos, o presente estudo observou que a maioria dos que apresentam DE, são SA e exibem escores mais baixos de qualidade de vida em todos os domínios.

Observa-se que a maioria dos idosos pesquisados nos grupos de convivência são $\mathrm{SA}$, sendo que entre os homens com DE, mais da metade pratica a quantidade de $\mathrm{AF}$ recomendada, que é de 150 minutos semanais ${ }^{20}$. Altos níveis de pratica de $\mathrm{AF}$ também foram encontrados entre idosos que participam de grupos de convivência em Florianópolis/SC ${ }^{25}$ e São José/SC, principalmente entre os homens ${ }^{26}$. Da mesma forma, quando categorizados de acordo com a severidade da doença, a maioria dos idosos classificou-se como SA, apesar de não apresentar diferença significativa.

A maior prevalência de AF entre os homens com DE nesse estudo pode ter relação com o fato destes idosos frequentarem grupos de convivência, onde geralmente são praticadas atividades físicas como dança e ginástica. A participação em grupos de convivência contribui para um estilo de vida mais ativo, favorecendo a adoção de hábitos saudáveis e proporcionando uma vida com mais autonomia e independência funcional ${ }^{27,28}$, o que consequentemente, melhora a $\mathrm{QV}$ e a condição emocional no envelhecimento.

A AF proporciona benefícios ao sistema cardiovascular, na redução de doenças vasculares e aumento do fluxo sanguíneo em determinados leitos vasculares ${ }^{9}$, sendo que tais efeitos podem auxiliar na vascularização peniana ${ }^{10}$. Segundo Pohjantahti-Maaroos, Palomaki e Hartikainen ${ }^{29}$, indivíduos que praticam AF regular (>400 kcal/dia) têm menor chance de apresentar DE, sendo que esse efeito positivo nos fatores de risco seria eficiente tanto para reverter como atenuar a $\mathrm{DE}^{4}$. Embora a amostra do estudo tenha apresentado nível suficiente de Atividade física, eles apresentaram maiores índices de sobrepeso, diabetes e hipertensão que são fatores associados à DE.

Os idosos com DE deste estudo apresentaram escores mais baixos de QV em todos os domínios, psicológico e social. A QV inferior apresentada no domínio social pode ter relação com o processo de envelhecimento vivenciado pelos idosos do presente estudo, pois o mesmo pode provocar uma série de mudanças; como o aparecimento de doenças crônicas, limitações físicas, perdas cognitivas, sintomas depressivos, declínio sensorial, acidentes e isolamento social ${ }^{30}$. Quando relacionada a QV à severidade da DE, os idosos sem DE apresentaram melhor qualidade de vida quando comparados aos com DE Severa e Moderada nos domínios Psicológico e Social. 
O presente estudo apresenta algumas limitações, dentre elas o desenho do estudo; caracterizado como transversal, pode impedir a determinação da relação temporal dos eventos. A obtenção de dados exclusivamente por questionário autorrelatado (IPAQ, WHOQOL e IIEF), no qual o relato dos sujeitos pode superestimar valores. A dificuldade de pesquisar com homens, por apresentarem-se em número reduzido quando comparados às mulheres. $\mathrm{E}$ ainda, pelo fato de a amostra ser exclusivamente composta por participantes de grupos de convivência, e entre essa população a prática de $\mathrm{AF}$ tende a ser maior do que população em geral, já que nesses grupos ocorrem rotineiramente AF diversas. Nesse contexto, cabe ressaltar a necessidade de novas pesquisas que avaliem os aspectos investigados neste estudo, estendendo-os a outras faixas-etárias e em diferentes contextos culturais e sociais, por tratar-se de um assunto delicado e ainda pouco explorado no universo masculino.

Através dos dados obtidos, conclui-se que houve alta prevalência de DE entre os idosos pesquisados, sendo a maioria severa. Os homens com DE apresentaram menores escores de QV nos domínio psicológico e social. A maioria dos homens tanto os com DE quanto sem DE foram classificados como SA fisicamente. Acredita-se que são necessárias novas pesquisas que relacionem a prática de $\mathrm{AF}, \mathrm{QV}$ e DE haja vista a grande importância desses fatores, já que a DE é um aspecto que pode alterar a percepção da QV masculina. Sendo um grande problema na saúde pública brasileira, alerta para a necessidade de prevenção masculina aos fatores de risco ao aparecimento da DE e possível redução dos seus sintomas, principalmente por meio da prática de AF.

\section{Referências}

1. Figueiredo W. Assistência à saúde dos homens: um desafio para os serviços de atenção primária. Cien Saude Colet. 2005; 10(1): 105-9.

2. Medeiros PA, Streit IA, Sandreschi PA, Fortunato AR, Mazo GZ. Participação masculina em modalidades de atividades físicas de um Programa para idosos: um estudo longitudinal. Cien Saude Colet. 2014; 19(8): 3479-3488.

3. Abdo CHN, Oliveira Jr WM, Scanavin MT, Martins FG. Disfunção Erétil - resultados do estudo da vida sexual do brasileiro. Rev Assoc Med Bras. 2006; 52(6): 424-29.

4. Souza CA, Cardoso FL, Silveira RA, Wittkopf PG. Importância do exercício físico no tratamento da disfunção erétil. Rev Bras Cardiol. 2011; 24(3): 180-5.

5. Zambon JP, Mendonça RR, Wroclawski ML, Karan Junior A, Santos RD, Carvalho JAM, et al. Cardiovascular and metabolic syndrome: risk among men with and without erectile dysfunction: case-control study. São Paulo MeD J. 2010; 128(3): 137-40.

6. Giami A, Nascimento KC, Russo J. Da impotência à disfunção erétil: destinos da medicalização da sexualidade. Physis. 2009; 19(3): 637-58.

7. Cheng, JYW, Ng EML. Body mass index, physical activity and erectile dysfunction: An U-shaped relationship from population-based study. Int J Obes. 2007; 31(10):1571-8.

8. Kalka D, Domagala Z, Dworak J, Womperski K, Rusiecki L, Marciniak W, et al. Association between physical exercise and quality of erection in men with ischaemic heart disease and erectile dysfunction subjected to physical training. Kardiologia Pol. 2013; 71(6): 573-80.

9. Posch K, Schmidt K, Graier WF. Selective stimulation of L-arginine uptake contributes to shear stress-induced formation of nitric oxide. Life sci. 1999; 64(8): 663-70.

10. Esposito K, Giugliano F, Di Palo C, Giugliano G, Marfella R, D’Andrea F, et al. Effect of lifestyle changes on erectile dysfunction in obese men: a randomized controlled trial. JAMA. 2004; 291(24): 2978-84.

11. Esposito K, Giugliano D. Lifestyle/dietary recommendations for erectile dysfunction and female sexual dysfunction. Urol Clin North Am. 2011; 38(3): 293-301.

12. Agostini LCM, Netto JMB, Miranda Junior MV, Figueiredo AA. Erectile dysfunction association with physical activity level and physical fitness in men aged $40-75$ years. Int J Impot Res. 2011; 23(3): 115-21. 
13. World Health Organization (WHO). AUDIT - The Alcohol Use Disorders Identification Test: Guidelines for Use in Primary Care (second edition), 2001. Disponível em: http:// whqlibdoc.who.int/hq/2001/ WHO_MSD_MSB_01.6a.pdf?ua=1. Acesso em: 21 de abril de 2015.

14. Moretti-Pires RO, Corradi-Webster CM. Adaptação e validação do Alcohol Use Disorder Identification Test (AUDIT) para população ribeirinha do interior da Amazonia, Brazil. Cad Saúde Pública. 2011; 27(3): 497-509.

15. World Health Organization (WHO). Body Mass Index classification, 2004. Disponível em: http://apps.who.int/bmi/index.jsp?introPage=intro_3.html. Acesso em: 21 de abril de 2015.

16. Instituto Brasileiro de Geografia e Estatística (IBGE). Censo demográfico, 2010. Disponível em: <http://www.ibge.gov.br/cidadesat/topwindow.htm?1> Acesso em: 16 de mai. 2013.

17. BenedettiTRB, Antunes PC, Rodriguez-Anez CR, Mazo GZ, PetroskiEL. Reprodutibilidade e validade do questionário internacional de atividade física (IPAQ) em homens idosos. Rev Bras Med Esporte. 2007; 13(1): 11-16.

18. Mazo GZ, Benedetti TRB. Adaptação do questionário internacional de atividade física para idosos. Rev Bras Cineantropom Desempenho Hum. 2010; 12(6): 480-84.

19. Fleck MPA, Louzada S, Xavier M, Chachamovich E, Vieira G, Santos L, et al. Aplicação da versão em português do instrumento abreviado de avaliação da qualidade de vida “WHOQOL-bref”. Rev Saúde Pública. 2000; 34(2): 178-83.

20. Rosen RC, Riley A, Wagner G, Osterloch IH, Kirkpatrick J, Mishra A. The international index of erectile function (iief): a multidimensional scale for assessment erectile dysfunction. Urology. 1997; 49(6): 822-30.

21. Santos PR. Disfunção erétil e Qualidade de vida em pacientes jovens submetidos à hemodiálise. J Bras Nefrol. 2008; 30(2): 132-6.

22. Naidan N, Rivaad OE, Muukhai N, Janlav M. Testosterone deficiency with erectile dysfunction in Mongolian men. World J Mens Health. 2013; 31(2): 170-5.

23. Ferraz BM, Ciconelli JRM. Tradução e adaptação do índice internacional de função erétil para a língua portuguesa. RBM - Revista Brasileira de Medicina. 1998; 55: 35-40.

24. Gonzáles AI, Sties SW, Wittkopf PG, Mara LS, Ulbrich AZ, Cardoso LF, et al. Validação do Índice Internacional de Função Erétil (IIFE) para Uso no Brasil. Arq Bras Cardiol. 2013; 101(2): 176-82.

25. Benedetti TRB, Mazo GZ, Borges LJ. Condições de saúde e nível de atividade física em idosos participantes e não participantes de grupos de convivência de Florianópolis. Cien Saude Colet. 2012; 17(8): 2087-93.

26. Cardoso AS, Levandoski G, Mazo GZ, Prado APM, Cardoso LS. Comparação do nível de atividade física em relação ao gênero de idosos participantes de grupos de convivência. RBCEH. 2008; 5(1): 9-18.

27. Almeida EA, Madeira GD, Arantes PMM, Alencar MA. Comparação da qualidade de vida entre idosos que participam e idosos que não participam de grupos de convivência na cidade de Itabira-MG. Rev Bras Geriatr Gerontol. 2010; 13(3): 435-43.

28. Wichmann FMA, Couto AN, Areosa SVC, Montanes MCM. Grupos de convivência como suporte ao idoso na melhoria da saúde. Rev Bras Geriatr Gerontol. 2013; 16(4): 821-32.

29. Pohjantahti-Maaroos H, Palomaki A, Hartikainen J. Erectile dysfunction, physical activity and metabolic syndrome: differences in markers of atherosclerosis. BMC Cardiovasc Disord. 2011; 11(36): 1-9.

30. Ramos LR. Fatores determinantes do envelhecimento saudável em idosos residentes em centro urbano: Projeto Epidoso, São Paulo. Cad. Saúde Pública. 2003; 19(3): 793-8.

ENDEREÇO PARA

CORRESPONDÊNCIA

CAMILA DA CRUZ RAMOS DE ARAUJO

cami.ramosdearaujo@hotmail.com
Rua Evaldo Schaeffer, 422, bloco B10,

Apto 23, Jardim Atlântico, Florianópolis,

SC, Brasil, 88095350. (48)99118140
RECEBIDO REVISADO APROVADO

$23 / 11 / 2015$ $12 / 02 / 2016$ $13 / 02 / 2016$ 